\title{
USO DE LA AUTOREVELACIÓN DEL PSICOTERAPEUTA LATINO, COMO HERRAMIENTA PARA MEJORAR LA RELACIÓN TERAPEUTICA
}

\author{
USE OF SELF-DISCLOSURE BY THE LATINO THERAPIST, AS A \\ TOOL TO IMPROVE THE THERAPEUTIC RELATIONSHIP
}

\author{
Silvia Sady-Kennedy Vaughn, Ph.D \\ Docente Carrera de Psicología - UPSE \\ doctoravaughn@yahoo.com
}

\section{RESUMEN}

El propósito de este estudio fue promover el uso de la autorevelación en terapia con pacientes Latinos en países Anglosajones. El estudio comparó la percepción y los valores del uso de la auto-revelación durante la sesión terapéutica, entre terapeutas Latinos y no-Latinos. Se utilizó un cuestionario de auto -administración enviado por correo electrónico. Las respuestas se codificaron y tabularon y se utilizaron pruebas estadísticas de una cola para probar la hipótesis nula. Se analizaron cinco hipótesis de investigación: Los terapeutas Latinos: I) Reportarán que utilizan la auto-revelación con más frecuencia que los terapeutas no Latinos. (II) Afirmarán que perciben que la auto-revelación mejora la relación cliente-terapeuta más de terapeutas no Latinos. (III) afirmarán que perciben que la autorevelación del terapeuta abre el diálogo cliente- terapeuta y el desarrollo de la comprensión cultural, más que con los terapeutas no Latinos. (IV) Declararán que perciben la autorevelación como una técnica que aumenta la implicación terapéutica, más de terapeutas no Latinos. (V) Percibirán la auto-revelación como parte de su cultura más que los terapeutas no Latinos. Los resultados verificaron las primeras cuatro hipótesis e indican una diferencia significativa en la percepción de la auto-revelación y sus efectos en la terapia entre los terapeutas Latinos y no Latinos. En general, los resultados indican la importancia de la auto-revelación como una herramienta para mejorar la relación y la terapia de continuación.

Palabras clave: Ciencias de comportamiento, Latino, Minorías y grupos étnicos, auto-revelación, relación terapéutica

\begin{abstract}
The purpose of this study is to promote the use of self-disclosure in therapy with Latino patients in Anglo-Saxon countries. The study compared Latino and non-Latino therapists' perception and values of their use of Self-disclosure during session. A self-administered questionnaire was sent via e-mail. The responses were coded and tabulated and One-tail statistical tests were used to test the null hypotheses. Five research hypotheses were analyzed, The Latino therapist will: (I) Report that they use self-disclosure more frequently than non-Latino therapists will. (II) State that they perceive that self-disclosure improves the client-therapist relationship more than non-Latino therapists will. (III) State that they perceive that therapist self-disclosure opens client-therapist dialogue and the development of cultural understanding more than non-Latino therapists will. (IV) State that they perceive therapist self-disclosure as a technique that increases therapeutic involvement more than non-Latino therapists will. (V) Tend to perceive self-disclosure as part of their culture more than non-Latino therapists will. The results verified the first four hypotheses indicating a significant difference in the perception of self-disclosure and its effects in therapy between Latino and non-Latino therapists. The overall the results indicate the importance of self-disclosure as a tool to improve the therapeutic relationship and therapy continuation.
\end{abstract}

Keywords: Behavioral sciences, Latino, Minority \& ethnic groups, Self-disclosure, Therapeutic relationship

Recibido: septiembre de 2014 Aprobado: noviembre de 2014 


\section{Introducción}

De acuerdo a McGoldrick, Giordano y Pierce (1, p.25) "la etnicidad es una realidad social, la cual requerirá que el terapeuta sea más culturalmente competente a medida que entramos en el siglo 21."

Tanto en los campos de la salud mental como en la terapia de parejas y familias, las ideas sobre las diferencias étnicas deben ser tomadas en cuenta. En las minorías étnicas de los Estados Unidos de Norteamérica los servicios que estas reciben son desiguales, al igual que la calidad de los mismos y su accesibilidad. Los servicios sociales muestran persistentemente diferencias y separación en las poblaciones de minorías diversas.

Los estudios indican que pocos servidores de salud mental tienen el respaldo de conocimiento cultural para proveer servicios a las minorías. Otros encuentran barreras de lenguaje y barreras en la forma en que el terapeuta se acerca al individuo o joining como describió el doctor Salvador Minuchin (2) en 1998.

Algunas minorías étnicas viviendo en Los Estados Unidos de Norteamérica, como por ejemplo los latinoamericanos, indican que dejan de concurrir a la terapia debido a la falta de

sensibilidad por parte de los profesionales. Estas características fueron descriptas por pacientes durante pasantías clínicas realizadas por este investigador, por lo cual se observaron las limitaciones de los terapeutas debido a la falta de competencia cultural y la influencia de los estereotipos sociales, que afectan negativamente el bienestar social.

Citado por Hastings (3), Szapocznik, Director del Centro de Estudios de la Familia de la Universidad de Miami, afirma: "La competencia cultural es una combinación de habilidad clínica y el conocimiento y la experiencia de una cultura particular. Cuando pienso en la competencia cultural, creo que no hay que ser solo un buen médico, sino también tener suficiente familiaridad con una cultura específica, y para poder asistir al proceso y la perspectiva de la familia y para ser capaces de identificar y se relacionarse con lo que son. Tienes que tener un poco de experiencia en ese ámbito para no perder nada."

Algunas instituciones tratan de considerar la raza, el género, la orientación sexual de los pacientes para disminuir los estereotipos, pero esto puede ser considerado como condescendiente para algunas minorías. (3)
El sistema de terapéutica o consejería se basa en un modelo euro americano y es considerado como sospechoso por muchas minorías. Algunos valores y asunciones son basadas en la civilización del Oeste. (4) Con esto en mente, muchos pacientes sienten que tienen derecho a saber sobre sus terapeutas, su historia y pasado, su origen, religión, y orientación sexual. Gladding, Remly y Huber, 2001 (5) determinaron que muchos terapeutas están de acuerdo en informar y clarificar sobre su propia identidad en la práctica.

Sue and Sue (4) dijeron que los terapeutas blancos son más pasivos y proveen menos información privada, indicando que "un paciente multicultural no se abrirá hasta que el terapeuta se autorevele primero."

¿Cómo se puede mostrar el entendimiento de la cultura cuando el efecto del privilegio del blanco está siempre presente?. Los pacientes de otras culturas se deben adaptar a la nueva sociedad, la cual no siempre está abierta para acomodar las necesidades de los recién llegados. El racismo y los estereotipos están presentes en la cultura de Norteamérica. Los estereotipos están presentes en la vida diaria, Neville et. al , 2001 (6) expresa que un terapeuta novato presenta un caso en supervisión y el mismo supervisor blanco le sugiere que todos los latinos son perezoso, ignorantes o violentos.

La necesidad de nuevas herramientas como la autorevelación auspician la confianza del Latino en el terapeuta, ya que el paciente se siente aceptado, la apreciación de las diferencias culturales y raciales mejora la confianza que el paciente tiene por el terapeuta. (7)

Cozby en 1973, (8) describe a la autorevelación como una técnica de comunicación en la cual el terapeuta da información en la sesión sobre el mismo para mejorar la relación terapéutica. De acuerdo a Gladding (5, p. 206) "los Terapeutas sistémicos mantienen la idea que todo proceso que se utiliza impacta más allá del individuo identificado". Por lo tanto, estos autores también mencionan que cualquier relación formada entre el terapeuta y el paciente va a producir cambios en su familia. Otros estudios como el de Peca-Baker \& Friedlander (9) indican que los terapeutas que autorevelan son clasificados favorablemente. Los latinos descubren muy rápidamente en el mundo del Anglo la soledad y la separación, para ellos la familia y la unión de la misma (1, p. 151) es uno de los valores más importantes, por lo tanto la falta de familia y la soledad llevan al paciente a sufrir problemas de salud mental. El concepto de personalismo se refiere al individuo siendo tratado 
como tal, no como uno más. (1) Los latinos son curiosos con respecto a otras personas, les gusta hacer preguntas, en la terapéutica occidental se indica que la responsabilidad de la conversación depende del paciente. En el caso del latino, muchos no hablan a menos que se les hagan preguntas y sus estilos son diferentes de la clase predominante, por lo que no habrá comunicación si se sigue estas reglas. (4)

Las organizaciones terapéuticas determinan los reglamentos que hacen al terapeuta honesto, pero que no están orientados a la diversidad cultural. Estos mandatos limitan la conexión del paciente y el terapeuta. Los paciente no entienden esta frialdad y el profesionalismo se malinterpreta.

\section{Revisión Literaria}

De acuerdo a Jourard (10, p 19) revelar significa dar a conocer, manifestar, o mostrar. Autorevelación es el acto de manifestarse uno mismo, y de mostrarse para que otros puedan percibirlo. Yalom (11), dice que la autorevelación varía dependiendo de la escuela de la que el terapeuta proviene, los psicoanalistas desaprueban y los humanistas la favorecen.

Behar (12) habla de la posición neutral de los antropólogos al igual que los tradicionalistas psicoanalistas, en la cual la autorevelación pondrá al terapeuta en una posición vulnerable. Los estudios muestran que los terapeutas eligen estar más cercanos a sus pacientes, ya que las barreras aumentan las jerarquías y separan la diada paciente -terapeuta. La literatura o antecedentes previos a este estudio indican que la autorevelación es una problemática.

\section{Límites, legalidad y ética}

Epstein and Simon (13) crearon un índice, Ilamado, de explotación (EI), como una medida de investigación, que mide posibles violaciones de límites en las sesiones terapéuticas, y da una predicción de la explotación del paciente. En 1992, estos autores, enviaron 2500 cuestionarios a psiquiatras de los Estados Unidos de Norteamérica, recibieron 532 de retorno.

Encontraron una correlación entre el El y la posibilidad de que ocurra esa explotación del paciente. Estos autores creen que la autorevelación del terapeuta lleva a una unión social íntima entre el paciente y el terapeuta que viola los límites terapéuticos.

En 1991, Simon(14) describió que la ruptura de los límites en terapia causa daño terapéutico y principalmente describió la relación sexual entre la diada paciente terapeuta.

Simon retrata a la autorevelación como la primera causa de esta violación de límites. En 1997

el mismo autor investigó los límites terapéuticos, en esta ocasión dijo que los límites promueven la alianza terapéutica. En ninguno de estos estudios el autor evaluó la etnicidad o diversidad cultural.

En 1998, Williams (15) habló de la ruptura de límites basados en la violación de los mismos y los juicios por mala práctica profesional, en especial de la conducta sexual indebida del terapeuta. Describió a la autorevelación como dañina vista desde la teoría psicoanalítica pero

explicó que esos términos no son aceptados por otras teorías, lo cual limita la terminología. Williams mencionó que el terapeuta debe pensar más abiertamente y no limitarse, ya que estas limitaciones afectan la práctica profesional.

\section{Similitudes de los pacientes}

Edwards and Murdock (16) describieron en su estudio evaluando 400 terapeutas, la mayoría utilizó algún tipo de autorevelación para aumentar las similitudes entre ellos y sus pacientes. Este estudio no encontró diferencias de género o culturales.

Gibson (17) utilizó pacientes en su estudio, a diferencia de otros estudios que utilizan estudiantes. Explicó que el mayor uso de autorevelación, se observó en los terapeutas que

trataron de acercarse al paciente y hacer sentir al mismo como un igual a través de las

semejanzas en su vida. Esta técnica de acercamiento provee la oportunidad al terapeuta de modelar para el paciente nuevos comportamientos.

\section{Percepción del entrevistado}

Jourard (11) mencionó que los pacientes aumentan la confianza en el terapeuta cuando éste autorevela. Murphy y Strong, (18) mostraron que los pacientes perciben al terapeuta de mejor manera cuando éste autorevela.

Nilsson, Strassberg y Bannon (19) evaluaron a estudiantes que indicaron que los terapeutas que autorevelaron se los evaluó más favorablemente.

Lundeen and Schuldt (20) también mostraron que los terapeutas que autorevelaron se evaluaron más favorablemente. 


\section{El impacto terapéutico}

Patterson (21), estableció que no hay mucha investigación sobre autorevelación. Stricker (22, p 277 ) indicó que la autorevelación es "un proceso en el cual el yo es revelado", lo cual se realiza en forma verbal o no verbal.

Drouin (23) en el 2000 habló de la autorevelación como produciendo relaciones terapéuticas positivas, sin utilizar cultura o etnicidad.

\section{Grupos, feminismo, etnicidad}

Lawson, Lawson y Rivers en 2001 (24, p.17) hablaron de la importancia de la autorevelación en consejería de grupos de dependencia química. Identificaron esta herramienta como valiosa, debemos recordar que el beneficio es para "el cliente " (paciente ) como dicen Lawson et. al .

Mahalik, Van Omer y Simi en el 2000 (25) hablaron de la práctica feminista describen a la necesidad de autorevelación para equiparar la necesidad de las mujeres oprimidas, para aumentar la modelación de nuevas conductas y facilitar el proceso terapéutico.

En 2001 López y Carrillo (26) explicaron que los latinos preguntan cosas personales, recomendaron que la autorevelación debe ser corta, y también la necesidad de fijar límites con

los pacientes.

Constantine y Swan en 2003 (27) evaluaron la autorevelación como una manera cultural y dijeron que la autorevelación sirve como modelo para que el paciente hable también en la sesión. Mahalik ( 25) discutió las importancia del contexto en la autorevelación para ser efectiva y rápida.

\section{Materiales y Métodos}

Todos los métodos utilizados en este estudio siguieron las guías éticas de la Asociación Americana de Psicólogos (APA) y la Asociación de Terapeutas de Parejas y Familias (AAMFT) y fueron aprobados por la comisión de repaso (IRB) de la Universidad Alliant Internacional (AIU). Los participantes fueron seleccionados a través de correos electrónicos obtenidos de directorios de La AAMFT, MFT, Asociaciones de Trabajadores Sociales y APA, de Orange County en el estado de California, la participación fue voluntaria, a pedido de IRB cada participante firmó un consentimiento en el cual se estableció el motivo del estudio, la duración y los procedimientos.

La muestra incluyó trabajadores de la salud mental de origen Latino y No Latinos de diversos Estados (California, Nueva York, Texas, Arizona, Nevada, y Florida). El motivo de la selección de la muestras se basó en los directorios de salud mental los cuáles se identifican como servicios a latinos.

Los candidatos de origen No Latino que participaron como grupo de control, fueron seleccionados de los mismos directorios, omitiendo apellidos de origen Latino. La etnicidad fue parte del cuestionario, para medir la variable demográfica. La meta de la investigación fue la obtención de 100 respuestas para la muestra. Se ofreció a los participantes recibir un resumen de los resultados de la misma. La investigación esperaba mínima reactividad de la población debido a la anonimidad del estudio.

Criterios de inclusión

Fueron enviados 600 cuestionarios, para compleción; los profesionales debían poseer, una maestría o doctorado en la materia. Los datos colectados fueron de 107 participares, 60 mujeres y 47 hombres de los cuales $61 \%$ estipularon ser doctores, 51 describiéndose como latinos y 41 caucáseos, 3 asiáticos y 9 de otra etnia. El rango de edades fue de 22 a 66 años de edad. Los test enviados incompletos fueron excluidos del análisis al igual que los enviados por terapeutas no licenciados.

Tabla 1: Porcentaje de distribución de género y etnicidad

\begin{tabular}{|l|ll|ll|}
\hline $\begin{array}{l}\text { Género del Tera- } \\
\text { peuta }\end{array}$ & HOMBRES & MUJERES \\
\hline Latino & $\begin{array}{l}55.3 \% \\
=26\end{array}$ & $\mathrm{n}$ & $\begin{array}{l}41.6 \% \\
=25\end{array}$ & $\mathrm{n}$ \\
\hline No Latino & $\begin{array}{l}44.7 \% \\
=21\end{array}$ & $\mathrm{n}$ & $\begin{array}{l}58.4 \% \\
=35\end{array}$ & $\mathrm{n}$ \\
\hline Total & $\mathrm{n}=47$ & $\mathrm{n}=60$ & \\
\hline
\end{tabular}

\section{Instrumento: Encuesta}

\section{Procedimiento}

El estudio incluyó tres (3) preguntas de encuesta de respuesta múltiple y cinco (5) preguntas con escala de Likert y un caso corto con dos (2) preguntas. Una vez que las respuestas se marcaron, la información se guardó automáticamente en el sitio web, survey monkey.com El investigador tabuló la información en el programa estadístico de Statistical Package for the Social Sciences (SPSS). 


\section{Análisis de los datos}

Como lo indicado por Sprenkle y Moon (28), un problema en los cuestionarios de auto reporte es la reactividad del encuestado pudiendo alterar los resultados. El investigador asumió que en este caso, debido a ser terapeutas estos actuarían en forma ética y honesta. Las frecuencias fueron documentadas, se midieron las medias y la desviación estándar de cada una de las respuestas. La correlación estadística fue utilizada para la relación entre auto revelación y la relación terapeuta-paciente; autorevelación y el entendimiento cultural; auto revelación y continuación terapéutica; autorevelación y cultura latina. La estadística inferencial se basó en comparar terapeutas Latinos y no Latinos con datos nominales y ordinales. La hipótesis nula utilizó los niveles de 0.1 a 0.5 como rechazo de la misma. Los cuestionarios y repuestas demográficas se utilizaron como medidas para operacionalizar la variable dependiente.

\section{Resultados}

Se recibieron 124 respuestas, de las cuales 107 encuestas estaban completas. La respuesta del total de 600 envíos fue de $20.6 \%$ del total. Se utilizó el coeficiente Cronbach's Alpha de fiabilidad para preguntas IV y VIII demostrando que el cuestionario era confiable con un resultado de r.6498.

La fiabilidad se demostró en la percepción de los terapeutas con respecto a la autorevelación para abrir la conversación y el entendimiento cultural, el mejoramiento de la continuidad terapéutica y la relación terapeuta paciente, y el uso de auto revelación como herramienta cultural incluidos en las preguntas II y III de la encuesta que indicó un coeficiente Alpha de valor: 05049.

Con respecto al uso de la autorevelación en la sesión, en la tabla 3 se muestra la estadística descriptiva del análisis y la frecuencia de distribución.

Tabla 3: Frecuencia de uso de Autorevelación

\begin{tabular}{|l|l|r|r|r|}
\hline & $\begin{array}{l}\text { FRECUEN- } \\
\text { CIA }\end{array}$ & $\begin{array}{l}\text { P O R C E N - } \\
\text { TAJE }\end{array}$ & $\begin{array}{l}\text { PORCEN- } \\
\text { TAJE VÁ- } \\
\text { LIDO }\end{array}$ & $\begin{array}{l}\text { PORCENTAJE } \\
\text { ACUMULATIVO }\end{array}$ \\
\hline NUNCA & $\begin{array}{l}\text { FRECUEN- } \\
\text { CIA }\end{array}$ & 6.5 & 6.5 & 6.5 \\
\hline $\begin{array}{l}\text { O C A S I O - } \\
\text { NAL- MENTE }\end{array}$ & $\begin{array}{l}\text { PORCEN- } \\
\text { TAJE }\end{array}$ & 74.8 & 74.8 & 74.8 \\
\hline $\begin{array}{l}\text { FRECUEN- } \\
\text { TE- MENTE }\end{array}$ & $\begin{array}{l}\text { PORCEN- } \\
\text { TAJE VÁ- } \\
\text { LIDO }\end{array}$ & 18.7 & 18.7 & 18.7 \\
\hline TOTAL & $\begin{array}{l}\text { PORCEN- } \\
\text { TAJE ACU- } \\
\text { MULATIVO }\end{array}$ & 100.0 & 100.0 & 100.0 \\
\hline
\end{tabular}

La percepción propia del terapeuta se observa en la tabla 4 en la cual los terapeutas pudieron elegir más de una variable.

Tabla 4: Efectos de Autorevelación en sesión

\begin{tabular}{|l|l|r|r|}
\hline & FRECUENCIA & PORCENTAJE & \multicolumn{2}{|l|}{$\begin{array}{l}\text { PORCENTAJE } \\
\text { VÁLIDO }\end{array}$} \\
\hline $\begin{array}{l}\text { Paciente autore- } \\
\text { vela más }\end{array}$ & 95 & 88.8 & 88.8 \\
\hline $\begin{array}{l}\text { Mejorará terapéu- } \\
\text { tica }\end{array}$ & 75 & 70.1 & 70.1 \\
\hline Abre diálogo la & 51 & 51.4 & 51.4 \\
\hline $\begin{array}{l}\text { Mejora } \\
\text { continuación }\end{array}$ & 57.7 & 47.7 \\
\hline $\begin{array}{l}\text { Es herramien- } \\
\text { ta cultural }\end{array}$ & 26 & 24.3 & 24.3 \\
\hline $\begin{array}{l}\text { No creo en la } \\
\text { Autorevelación }\end{array}$ & 6 & 5.6 & 5.6 \\
\hline
\end{tabular}

Otros métodos utilizados fueron el Test Fisher de Probabilidad exacta para verificar el uso de autorevelación en terapeutas Latinos y No Latinos el cual dio un resultado significativo estadístico menor de $\mathrm{p} .<5$ de la primera a la cuarta hipótesis. Los resultados verificaron 4 de las 5 hipótesis del estudio. Demostrando que existe una correlación entre la etnia del terapeuta y el interés de autorevelación para promover la relación terapéutica, a pesar que los terapeutas Latinos no percibieron significativamente que la autorevelación, se debe a sus antecedentes culturales, ellos aceptan que la autorevelación es efectiva en abrir el diálogo y el entendimiento cultural.

\section{Discusión}

El investigador hipotetizó que la autorevelación es una herramienta valiosa para mejorar la relación terapéutica, abrir el diálogo cultural y mantener al paciente latino en la terapia. Los resultados indican que los terapeutas creen que la autorevelación mejora la relación terapéutica, abre el diálogo y mejora el entendimiento cultural, a la vez mejorando la continuidad del paciente en terapia.

Desde el punto de vista terapéutico, los pacientes se acercan más al terapeuta después que el mismo utiliza la autorevelación. La última hipótesis no fue comprobada ya que los terapeutas latinos no consideran a la autorevelación como un elemento cultural.

Comparando con otros estudios como Murphy y Strong (18), Simon (14), Constantine (27), los resultados han sido bastante consistentes, estos estudios indican que la autorevelación del terapeuta induce a que el paciente también autorevela su historia más efectivamente. Los instrumentos utilizados poseen buena validez interna y fiabilidad. 
Las limitaciones del estudio indican que la encuesta puede tener problemas de replicabilidad debido a problemas con validez externa, como lo indicado por Issac and Michael (29) .

El investigador del presente trabajo propuso que la última hipótesis no se verificó debido a lo siguiente:

- Algunos de los encuestados no se identificaron por su país de origen o etnia debido a que el formulario no incluía separación de países Latinoamericanos.

- Terapeutas Latinos criados en Los Estados Unidos de Norteamérica, no responden de la misma manera que si estuvieran en su país de origen.

- Existe un factor de aculturación, la adaptación a la nueva cultura cambia la perspectiva de las creencias, Sue \& Sue (3). Se supone que por ello el terapeuta Latino presentó ambivalencia ante la autorevelación debido a no querer quebrar la ética profesional, dejando su cultura de lado.

\section{Conclusiones}

Clínicamente este estudio sugiere que los terapeutas No Latinos deben tratar de entender la autorevelación como una herramienta que ayuda familiarizarse con el paciente y que lo mantiene en la terapia. También sugiere al terapeuta no Latino a comprender el sentido de las preguntas personales de los pacientes, no como una falta de respeto o quiebre de límites o que lo llevaran a involucrarse extremadamente con el paciente, sino como una terapia más personalizada, debido a esto los Latinos necesitan este tipo de terapia. Otra sugerencia es el entendimiento de la autorevelación como un elemento no intimidante, que ayuda a disminuir el distanciamiento y la jerarquía entre terapeuta y paciente. Será beneficioso para los terapeutas no latinos de explorar la posibilidad de proveer información sobre ellos mismos a sus pacientes latinos, sobre su etnicidad, cultura y profesión, en la primera entrevista, esto ayudará a disminuir la ansiedad de los pacientes y abrir el diálogo y mejorar el entendimiento cultural. El uso de la autorevelación debe quedar restringido para su uso únicamente para beneficiar al paciente, esto llevará a cambios en la visión ética sobre la autorevelación, si es utilizada con mente abierta como una técnica basada en la necesidad de la población.

\section{Recomendaciones}

Para futuros trabajos relacionados a la Auto- revelación, o trabajos de replicación de este estudio, extender el proceso en Latinoamérica, para observar, comparar y proveer una visión amplia del contexto cultural con su clasificación por país de origen.

Si los estudios se realizaran en países de Norteamérica o Europa, incluir la aculturación del terapeuta latino a ese país y su influencia en la Autorevelación.

\section{Referencias Bibliográficas}

1) Mc Goldrick M, Giordano J, Pierce, JK. Ethnicity and Family Therapy, (2da. ed) new

York: The Guilford Press; 1996.

2) Minuchin S,Nichols M. Family healing. New York: The Free Press; 1993.

3) Hastings C. How do you become culturally competent? Family Therapy Magazine, MarchApril, 2002; 18-24.

4) Sue DW, \& Sue, D. Counseling the culturally Diverse (4th ed) New York: John Wiley \& Sons, Inc. 2003

5) Gladding, S.T., Remley, T. P., \& Huber, C. H. Ethical, legal and professional issues in the practice of marriage and family therapy (3rd ed). Upper Saddle River, New Jersey: Merrill

Prentice Hall; 2001.

6) Neville, H.A:, Worthington, R.L:, \& Spanierman, L.B. .Race, power and multicultural counseling psychology. In Ponteroto, J. Casas, J. M., Suzuki, L.,\& Alexander, C. (Eds), Handbook of Multicultural Counseling (2.57-87) New York: Sage Public actions, Inc. (1991)

7) Helms, J.E.,\& Cook, D.A. Using race culture in counseling and psychotherapy: Theory and process. Boston: Allyn and Bacon; 1999.

8) Cozby, P.C. Self-Disclosure : a literature review. Psychological Bulletin, 1973, 79(2): 73-91

9) Peca-Baker, T.A., \& Friedlander,M.L. .Effects on role expectations on clients perceptions of disclosing and non-disclosing counselors. J COUNS DEV , 1987; 66, 78-81.

10) Jourard, S. M. Self-disclosure: an experimental analysis of the transparent self. New York: Wiley-Interscience; 1971

11) Yalom , I. The Theory and Practice of Group Psychotherapy (Third Ed). Basic Books, Inc; 1985.

12) Behar, $R$ The vulnerable observer. Boston: Beacon Press; 1996.

13) Epstein , R, S., Simon, R.I. Assessing boundary violations in psychotherapy: Survey results with exploitation index. Bulletin of the Menninger Clinic. 1992; 56(2), 150-67.

14) Simon, J. C. Criteria for therapist's self-disclosure. AM J PSYCHOTHER, 1988 XLII ( 3) 


\section{4- 15.}

15) Williams, M.H. Boundary Violation: The inappropriate lethal weapon of plaintiff's attorneys in psychotherapy malpractice lawsuits; 1998. In expertpages.com The original and leading directory of expert witnesses and consultants. [Recuperado October 1, 2004, de

Expertpages WebSite: http.//expertpages.com].

16) Edwards C. E.,\& Murdock, N.L. Characteristics of therapists self-disclosure in the counseling process. J Couns Dev. 1994; 72 (4): 384-9.

17) Gibson, D.L. Effects of therapist's self-disclosure on client's level of anxiety, perceptions of the therapist, and perceptions of the similarity to the therapist. Unpublished doctoral dissertation, CSPP; 1996

18) Murphy K.C. \& Strong, S.R. Some effects of similarity self-disclosure. JCOUNS PSYCHOL,1972; 19(2): 121-4.

19) Nilsson D. E., Strassberg, D.S.,\& Bannon,J. .Perceptions of counselor self-disclosure: an analogue study; J Couns Psychol. 1979; 26(5), (3994)

20) Lundeen, E. J.,\& Schuldt, W.J. Models of self-disclosure in psychotherapy. Psychology : A journal of Human Behavior. 1992; 29(2): 8-13. 